EPJ Web of Conferences 53, 04002 (2013)

DOI: $10.1051 /$ epjconf/20135304002

(C) Owned by the authors, published by EDP Sciences, 2013

\title{
Measurement of the energy spectrum of cosmic rays at the highest energies using data from Pierre Auger Observatory
}

\author{
Ioana C. Mariş ${ }^{1, a}$ for the Pierre Auger Collaboration²,b \\ ${ }^{1}$ LPNHE, 4 place Jussieu, 75252 Paris Cedex 05, France \\ ${ }^{2}$ Observatorio Pierre Auger, Av. San Martín Norte 304, 5613 Malargüe, Argentina \\ (Full author list: http://www.auger.org/archive/authors_2012_06.html)
}

\begin{abstract}
We report a measurement of the flux of cosmic rays with unprecedented precision and statistics using data from the Pierre Auger Observatory. Based on fluorescence observations in coincidence with at least one station of the surface detector we derive a spectrum for energies above $10^{18} \mathrm{eV}$. We also report on the energy spectra obtained with the surface detector array. The spectral features are presented in detail and the impact of systematic uncertainties on these features are addressed.
\end{abstract}

\section{INTRODUCTION}

The propagation and the origin of cosmic rays influence the number of particles that enter the Earth's atmosphere. The evolution of the flux with energy exhibits at the highest energies two features: the ankle, a flattening of the flux at about $4 \times 10^{18} \mathrm{eV}$, and a strong suppression above $5 \times 10^{19} \mathrm{eV}$. Currently it is not clear if these features are caused by the interaction of the particles with the cosmic background, or if they are hints of changes in the acceleration origin or power [1-4]. A precise measurement of the flux of cosmic rays is an important ingredient in discriminating between theoretical models.

The Pierre Auger Observatory is designed as an hybrid detector: a surface detector (SD) with more than 1600 water-Cherenkov stations placed on a triangular grid covering $3000 \mathrm{~km}^{2}$ and a fluorescence detector (FD) made of 27 optical telescopes grouped in five buildings, observing the atmosphere above the array. The detailed description of the Observatory can be found in [5-7]. The hybrid design allows for the energy calibration of the SD data and for obtaining the energy spectrum over two decades in energy. We present the measurements of the flux of cosmic rays above $10^{18} \mathrm{eV}$.

\section{AIR-SHOWERS RECONSTRUCTION}

The FD observes the fluorescence and Cherenkov light produced by the secondary particles from the air-showers in their passage through the atmosphere. The arrival direction is obtained from the timing information of the signal in individual triggered pixels and from at least one station of the SD. This set of events are referred to as hybrid events. From the reconstruction of the longitudinal profiles [8] the energy of the primary particle is inferred in an almost calorimetric way. The invisible energy, the fraction of energy carried by the muons and neutrinos, is obtained from parametrization of simulations assuming a mixed composition and QGSJet01 as hadronic interaction model. It is less than $10 \%$ above $10^{18} \mathrm{eV}$. The energy resolution is $7.6 \%$ [9].

\footnotetext{
ae-mail: Ioana.Maris@lpnhe.in2p3.fr

${ }^{\mathrm{b}}$ For the full authorlist see Appendix "Collaborations" in this volume.
}

This is an Open Access article distributed under the terms of the Creative Commons Attribution License 2.0, which permits unrestricted use, distribution, and reproduction in any medium, provided the original work is properly cited. 

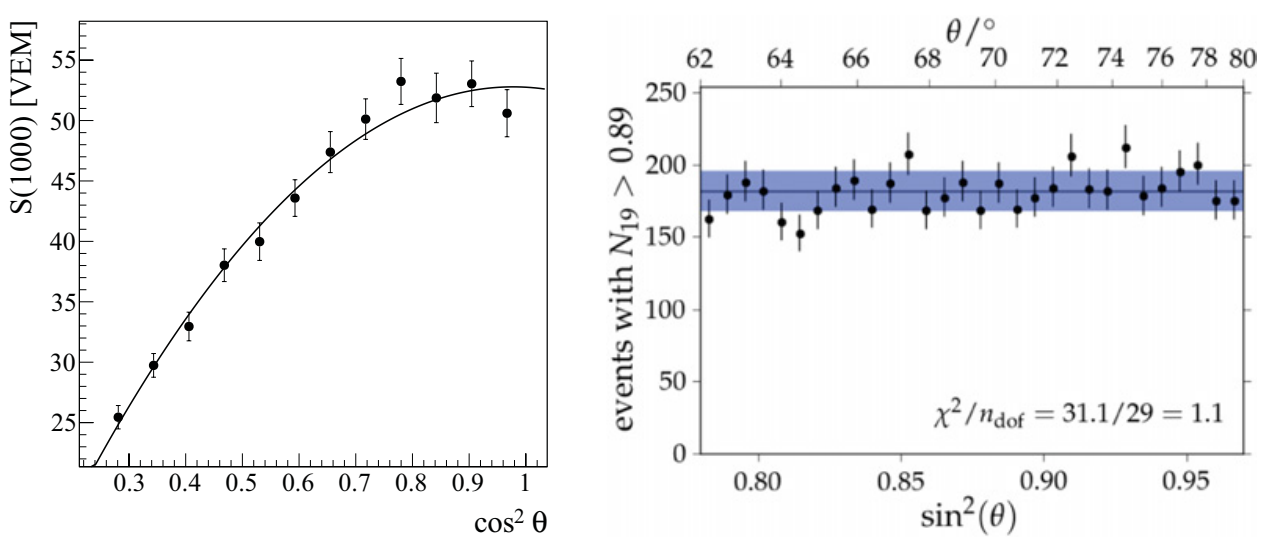

Figure 1. Left: $\mathrm{S}(1000)$ as a function of the zenith angle. Right: the number of events above $N_{19}=0.89$ as a function of the zenith angle.
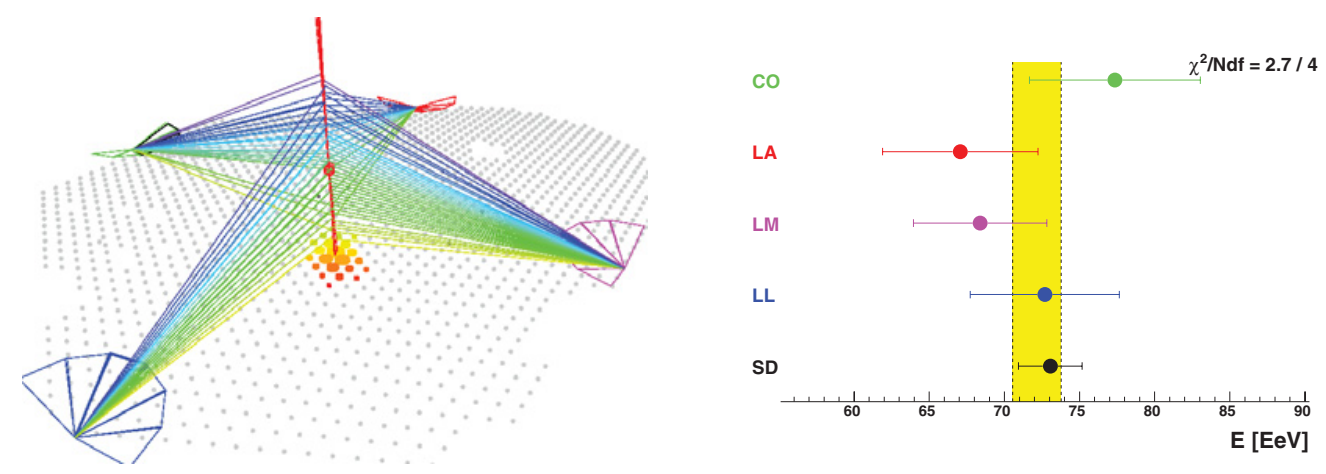

Figure 2. Example of an event with an energy of $7 \times 10^{19} \mathrm{eV}$ falling in the middle of the surface detector and observed with at least one telescope from each of the FD buildings (left); (right) the independent energy assignments from the four eyes (CO, LA, LM, LL) and from the surface detector (SD).

The signal from the particles reaching the ground is sampled with the water-Cherenkov detectors. The content of the air-showers at this level depends on the amount of matter traversed by the cascade. For vertical events(zenith angle smaller than 60 degrees) the signal contains contributions from both the electromagnetic and muonic components of the air-showers. At angles above 62 degrees (inclined events), the electromagnetic component is suppressed. This effect leads to different reconstruction methods for vertical and inclined events. In the first case the signal at $1000 \mathrm{~m}$ from the air-shower axis, $\mathrm{S}(1000)$, is interpolated from a lateral distribution function. $\mathrm{S}(1000)$ is corrected for the attenuation in the atmosphere through a constant intensity method: an air-shower with a zenith angle of 60 degrees produces a signal smaller by a factor of two then if it would have had an arrival direction of zero degrees (see Fig. 1 (left)). The energy estimator for the vertical events is $S_{38}$, the equivalent signal for a zenith angle of 38 degrees $[11,12]$. The reconstruction of the inclined events is based on a detailed modeling of the two dimensional muon density on the ground [13]. The energy estimator, $N_{19}$ is obtained by taking as a reference the normalization of the footprint of proton initiated air-showers with an energy of $10^{19} \mathrm{eV}$ and it is independent of the zenith angle (Fig. 1(right)).

An event example with an energy of $7 \times 10^{19} \mathrm{eV}$ is shown in Fig. 2. The air-shower has triggered at least one telescope in each FD buildings and the surface detector. The energies reconstructed independently with the data from the four eyes are in a very good agreement, even if the air-shower 


\section{UHECR 2012}
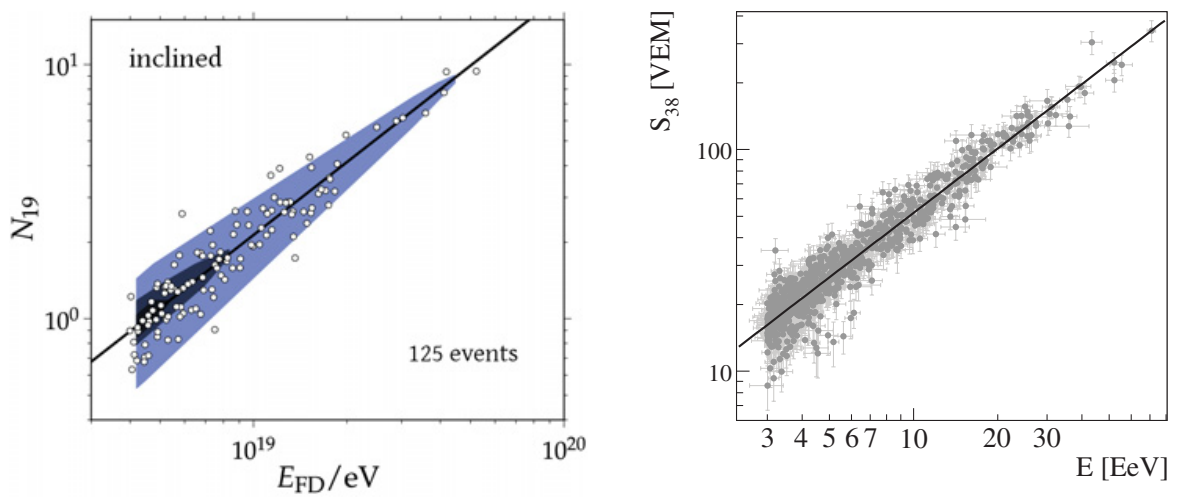

Figure 3. The energy calibrations curves for the inclined events (left) and for the vertical events (right). The lines represent the best fit to data.

is more than $20 \mathrm{~km}$ away. The energy measured from the SD data agrees with the FD reconstructions. The details of how we measure the energy with the SD are given next.

Energy calibration and resolution. To convert $N_{19}$ and $S_{38}$ to energy we use golden hybrid events: a sub-sample of the hybrid events which are independently triggered and reconstructed with the SD. We are selecting high quality longitudinal profiles, observed during a time period with clear atmosphere [10], with a cloud coverage of less than $25 \%$. Deeply penetrating air-showers would produce a larger signal on the ground that shallow ones. Thus, in order to reduce a possible mass composition bias we apply strict cuts on the fiducial field of view which assure an equal hybrid trigger probability for different primaries.

The correlation functions are obtained by maximizing the likelihoods and are in both cases almost linear $[11,14]$ :

$$
\begin{aligned}
& E_{\text {inclined }}=(4.69 \pm 0.09) \times 10^{18} \mathrm{eV} \cdot N_{19}^{(1.05 \pm 0.02)}, \\
& E_{\text {vertical }}=(1.68 \pm 0.05) \times 10^{17} \mathrm{eV} \cdot S_{38}^{(1.035 \pm 0.009)} .
\end{aligned}
$$

The energy calibrations, shown in Fig. 3, induce a systematic uncertainty on the energy due to the limited statistics: 125 events for the inclined data and 839 for the vertical data. Namely $7 \%$ at $10^{19} \mathrm{eV}$ and $12 \%$ at $10^{20} \mathrm{eV}$ for the vertical events and $13 \%$ at $10^{19} \mathrm{eV}$ and more than $50 \%$ at $10^{20} \mathrm{eV}$ for the inclined events. In the future with more accumulated data, these uncertainties will be reduced. Assuming a constant energy resolution for the hybrid energy of $\approx 8 \%$, the energy resolution for the vertical data set can be obtained from the golden hybrid events (Fig. 4). The resulting SD energy resolution decreases from $\sigma_{E} / E_{\text {vertical }}=(15.8 \pm 0.9) \%$ below $6 \times 10^{18} \mathrm{eV}$ to $(12.0 \pm 1.0) \%$ for $E_{\mathrm{SD}}>10^{19} \mathrm{eV}$, the reconstruction uncertainties being important at $3 \times 10^{18} \mathrm{eV}$, while at the highest energies the showerto-shower fluctuations of $\mathrm{S}(1000)$ are dominant.

The three measurements with inclined, vertical and hybrid events inherit the same systematic uncertainty on the energy from the fluorescence detector energy assignment. The determination of the absolute value of the fluorescence yield contributes with $14 \%$, the reconstruction of the energy with $4 \%$ due to the invisible energy correction, and $10 \%$ from the reconstruction of the longitudinal profiles, while the atmospheric effects add another $8 \%$. The total systematic uncertainty of $22 \%$, includes as well the calibration of the detector, and the propagated uncertainties of the aerosol optical depth measurements [11]. 

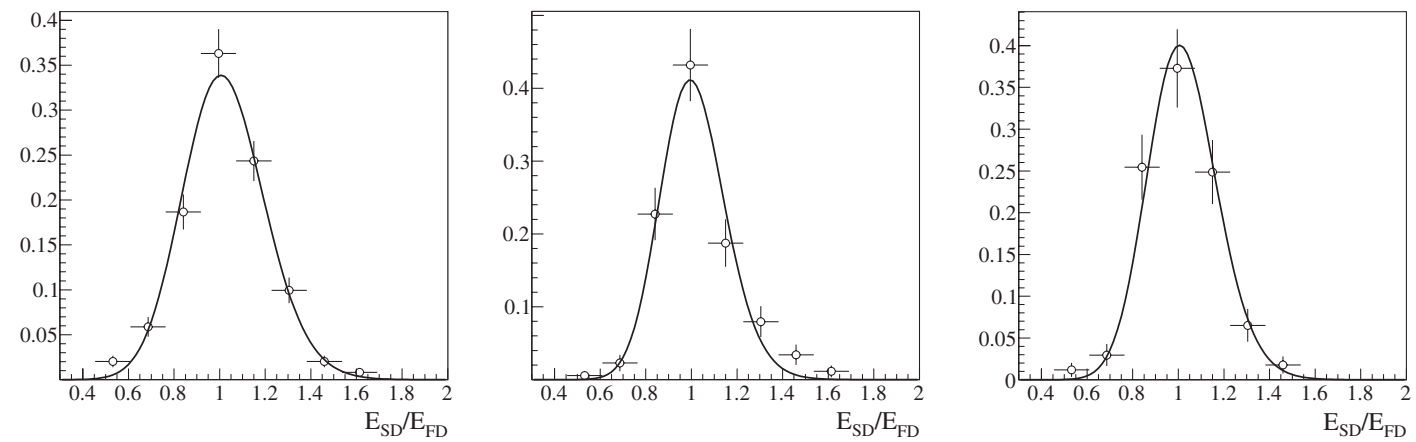

Figure 4. The ratio between the energies reconstructed with the hybrid and the surface detector data for different energy intervals: between $3 \times 10^{18} \mathrm{eV}$ and $6 \times 10^{18} \mathrm{eV}$ (left) between $6 \times 10^{18} \mathrm{eV}$ and $10^{19} \mathrm{eV}$ (center) and above $10^{19} \mathrm{eV}$ (right).
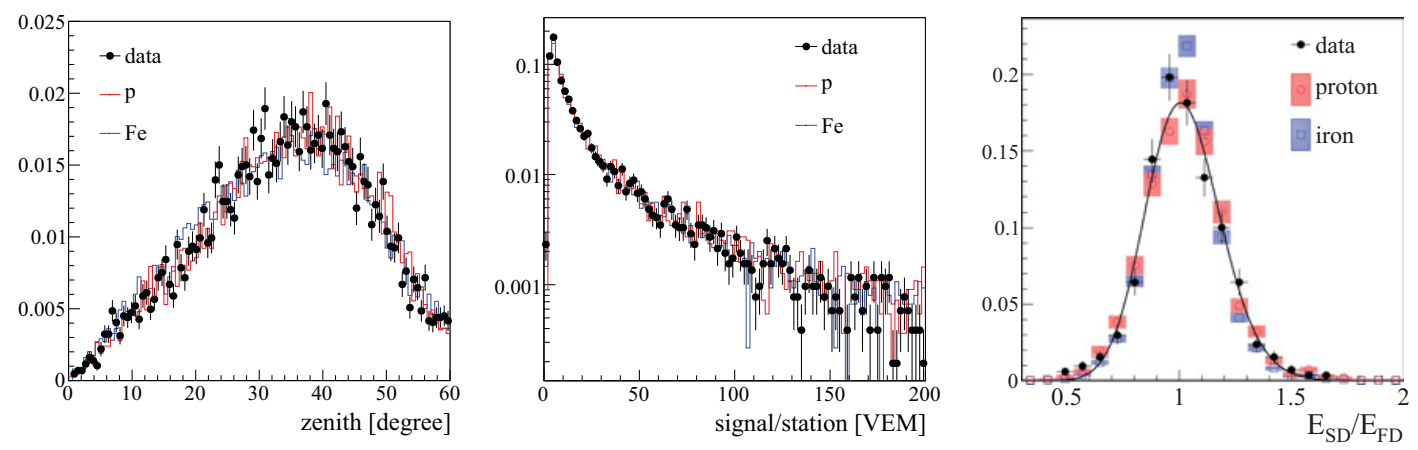

Figure 5. Comparison between data and MC. The distributions are shown for QGSJet II.03 for proton and iron. Simulations have been rescaled to take into account the missing number of muons. (left) Normalized distribution of the number of events as a function of the zenith angle; (center) Normalized distribution of the signal in individual stations for all the stations in the recorded and simulated events; (right) Normalized distribution of the FD and SD energy ratio.

\section{ENERGY SPECTRUM}

The energy resolution of the surface detector is energy dependent and thus it distorts the distribution of events as a function of energy by bin-to-bin migrations. To obtain the true number of events a forwardfolding procedure is used. The migration matrices are built from air-showers and detector simulations by using CORSIKA [15] with QGSJet II.03 [16] as hadronic interaction model. Even if the number of muons is underestimated in the simulations [17], after an energy rescaling such that for the same true $S_{38}$ we obtain the same energy in data and simulations, the distributions in the main shower observables are reproduced by the simulations: in Fig. 5 are depicted the normalized distribution for events with an energy above $3 \times 10^{18} \mathrm{eV}$ of the zenith angle, of the signal per station including all stations in the events and of the ratio of the energy determinations with the FD and SD, for proton and iron primaries.

The exposure calculation for the surface detector is based on the time integration of the instantaneous effective area [18]. It amounts up to end of 2010 to $20905 \mathrm{~km}^{2}$ sr year for the vertical data, while for the inclined events it is four times smaller due to the reduced solid angle considered (zenith angle between 62 and 80 degrees). The SD energy spectra $[11,14]$ are deduced only in the energy range with full trigger efficiency, i.e. $3 \times 10^{18} \mathrm{eV}$ for the vertical events and $4 \times 10^{18} \mathrm{eV}$ for the inclined events. The enhancement of the surface detector with a denser array [19] (750 $\mathrm{m}$ spacing between detectors) has 


\section{UHECR 2012}

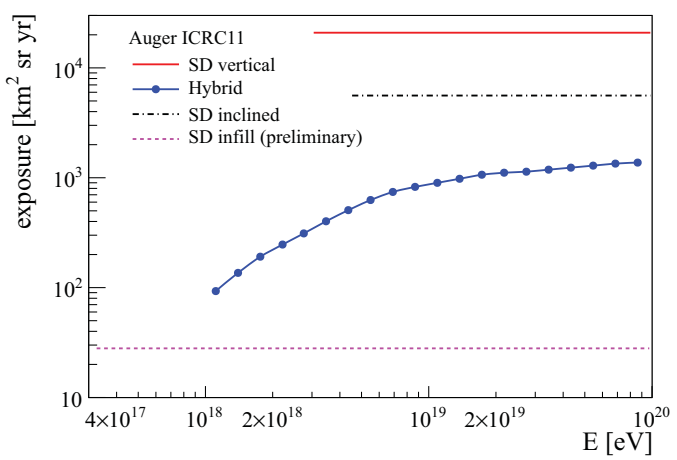

Figure 6. Exposures for the vertical and inclined SD measurements together with the hybrid one. The SD exposures are illustrated only for the energy range of full trigger efficiencies. The exposure for the $750 \mathrm{~m}$ spacing array (infill) is also shown.

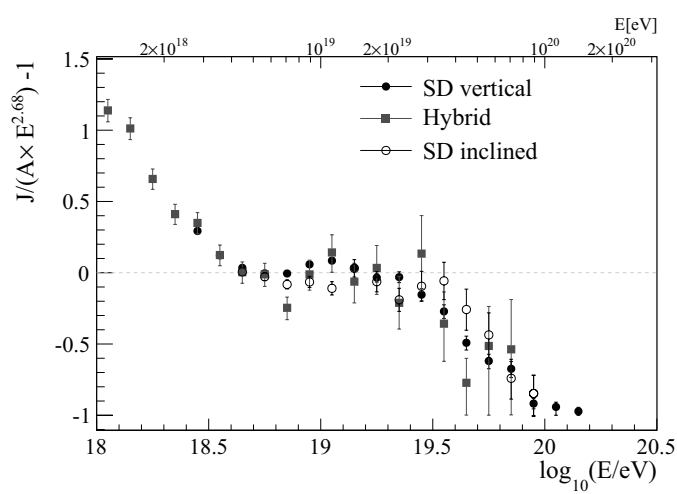

Figure 7. The energy spectra measured with the data recorded at the Pierre Auger Observatory. All the three measurements are in a very good agreement in the energy range of overlap.

been completed. It will increase the energy range for the SD by one decade. The integrated exposure for the same period amounts to $29 \mathrm{~km}^{2} \mathrm{sr}$ year [20]. In case of the hybrid measurement the exposure calculation is based on detailed simulations of the detector [21], taking into account its time evolution. The simulations consider, besides the SD and FD hardware status every 10 minutes, all the atmosphere measurements and monitoring data. The lower energy limit is given by the trigger probability of the SD: above $10^{18} \mathrm{eV}$ all events with a zenith angle of less than 60 degrees produce a signal in at least one SD station [22]. The exposures are represented in Fig. 6, while the energy spectra obtained with the three data sets are illustrated in Fig. 7. The agreement between the energy spectra is very good, the difference in fluxes is less than 5\%. All the energy spectra share the same systematic uncertainty on the energy, $22 \%$, due to the cross-calisbration of the surface detector data with the hybrid energy determination. The systematic uncertainties caused by the exposure determination and energy resolution effects, are independent. For the hybrid measurement they vary from $10 \%$ at $10^{18} \mathrm{eV}$ to $6 \%$ at $>10^{19} \mathrm{eV}$. In the case of the vertical surface detector they are 6\% (3\% exposure determination and 5\% from the unfolding of the energy resolution effects) while for the inclined spectrum the exposure contributes with a similar systematic uncertainties. Taking into account the independent systematic uncertainties of the hybrid and vertical SD measurements we obtain an energy spectrum which extends over the whole energy range above $10^{18} \mathrm{eV}$ (Fig. 8). The inclined spectrum has not been yet deconvoluted for the energy resolution effects and thus not included in the combination. To determine the changes in 


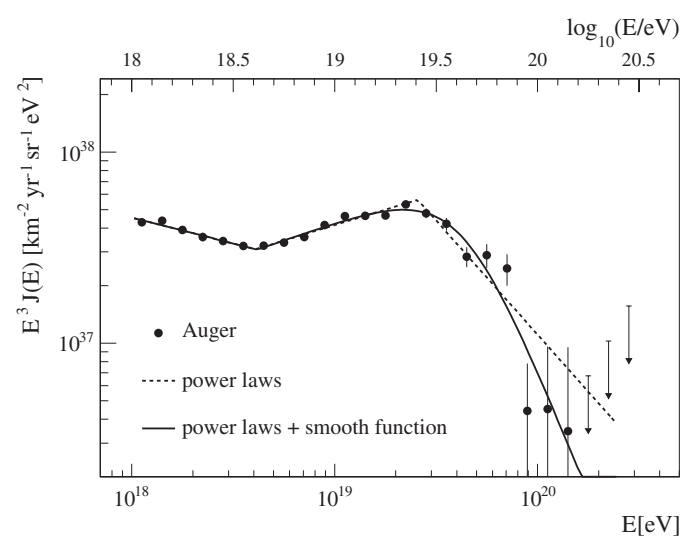

Figure 8. The features of the Auger energy spectrum as obtained from fits with two functions (see text). The systematic uncertainties on the energy mount up to $22 \%$ and are not shown in the graph.

the flux evolution with energy we fit two functions: three power laws with free breaks and two power laws with a sigmoid suppression. The spectral index changes from $(-3.27 \pm 0.02)$ to $(-2.68 \pm 0.01)$ at $\log _{10}(E / \mathrm{eV})=18.61 \pm 0.01$ and then to $(-4.2 \pm 0.1)$ at $\log _{10}(E / \mathrm{eV})=19.41 \pm 0.02$.

\section{CONCLUSIONS}

The flux of cosmic rays with energies above $10^{18} \mathrm{eV}$ has been measured with the data of the Pierre Auger Observatory, with three independent methods. The agreement between the energy spectra in the regions of overlap is better than $5 \%$, well within the independent systematic uncertainties. The change in the spectral index at $(4.1 \pm 0.2) \times 10^{18} \mathrm{eV}$, the ankle, and the flux suppression starting at $(2.6 \pm 0.2) \times 10^{19} \mathrm{eV}$ have been measured with very large statistics. The current measurements are dominated by the systematic uncertainties on the energy, 22\%. To achieve a more accurate measurement of the spectral features the systematic uncertainties on the energy, mostly due to the fluorescence yield and reconstruction, will be reduced. The extension of the array with the denser sub-detector will allow the measurement of the spectral slope down to $4 \times 10^{17} \mathrm{eV}$ and the overlap with the KASCADE-Grande measurement [24]. A further enhancement to even lower energies, with an array of $350 \mathrm{~m}$ spacing, is foreseen. We have measured 77 events above $5 \times 10^{19} \mathrm{eV}$ in the vertical surface detector data set, corresponding to about 4 years of full detector efficiency. In order to accumulate more statistics at the highest energies, a continuation of the data taking and of the detector maintenance is essential.

\section{References}

[1] V. Berezinsky, A. Z. Gazizov, and S. I. Grigorieva, Phys. Lett. B 612 (2005) 147

[2] A. M. Hillas, J. Phys. G31 (2005) R95

[3] T. Wibig and A. W. Wolfendale, J. Phys., G 31 (2005) 255

[4] D. Allard et al., JCAP 0810 (2008) 033

[5] The Pierre Auger Collaboration, Nucl. Instr. Meth. A 523 (2004) 50

[6] I. Allekotte et al., Nucl. Instr. and Meth. A 586 (2008) 409

[7] The Pierre Auger Collaboration, Nucl. Instr. and Meth. A 620 (2010) 227

[8] M. Unger et al., Nucl. Instr. Meth. A 588 (2008) 433

[9] F. Salamida for the Pierre Auger Collaboration, Proc. 32nd ICRC (2011), arXiv:1107.4809v1

[10] The Pierre Auger Collaboration, Astropart. Phys. 33 (2010) 108

[11] R. Pesce for the Pierre Auger Collaboration, Proc. 32nd ICRC (2011), arXiv:1107.4809v1 


\section{UHECR 2012}

[12] The Pierre Auger Collaboration, Phys. Rev. Lett. 101 (2008) 061101

[13] G. Rodriguez for the Pierre Auger Collaboration, these proceedings

[14] H. Dembinski for the Pierre Auger Collaboration, Proc. 32nd ICRC (2011), arXiv:1107.4809v1

[15] D. Heck et al. Report FZKA 6019, Forschungszentrum Karlsruhe (1998)

[16] S. Ostapchenko, Nucl. Phys. B (Proc. Suppl.) 151 (2006) 143

[17] A. Yushkov for the Pierre Auger Collaboration, these proceedings

[18] The Pierre Auger Collaboration, Nucl. Instr. and Meth. A 613 (2010) 29

[19] F. Sanchez for the Pierre Auger Collaboration, Proc. 32nd ICRC (2011), arXiv:1107.4807v1

[20] I. C. Mariş for the Pierre Auger Collaboration, Proc. 32nd ICRC (2011), arXiv:1107.4809v1

[21] The Pierre Auger Collaboration, Astropart. Phys. 34 (2011) 368

[22] The Pierre Auger Collaboration, Astropart. Phys. 35 (2011) 266

[23] The Pierre Auger Collaboration, Phys. Lett. B 685 (2010) 239

[24] The KASCADE-Grande Collaboration, Astropart. Phys. 36 (2012) 183 